\title{
Mesothelioma in the age of "Omics": Before and after The Cancer Genome Atlas
}

\author{
David T. Severson, DPhil, Assunta De Rienzo, PhD, and Raphael Bueno, MD
}

Supplemental material is available online.

Feature Editor's Introduction-Malignant pleural mesothelioma (MPM) is a highly fatal cancer of the pleura that has been defeating standard and investigational therapies since its first description. The efficacies of chemotherapy, radiotherapy, and surgical therapy are limited, and we have been writing for decades that improved therapies are needed. MPM is born of inflammation, and approximately $80 \%$ of cases are associated with the smoldering tissue inflammatory responses against the carcinogenic fibers of asbestos. Emerging data on the use of programmed cell death protein 1 immune checkpoint inhibitors were initially exciting, but response is less than $20 \%$ and these agents are finding their place on the list of approaches with narrow efficacy. Molecular targeted therapies have revolutionized the treatment of other cancers, commonly result in striking antitumor responses, and directly embody precision medicine. For an example, we prescribe drugs for some lung adenocarcinomas that target the secondary mutations that develop as a resistance mechanism to their initial targeted therapy. The discovery of molecular therapeutics for any tumor begins with identification of a target through investigation of the genomic, epigenomic, and transcriptomic drivers of its carcinogenesis. Such an advance could revolutionize the treatment of mesothelioma. A comprehensive dissection of MPM's molecular structure was recently published by 2 groups, the first from the Brigham and Women's Hospital and then from The Cancer Genome Atlas. In the Invited Expert Opinion article that follows, a practical account of the molecular underpinnings of

From the Thoracic Surgery Oncology Laboratory and the International Mesothelioma Program (www.impmeso.org), Division of Thoracic Surgery and the Lung Center, Brigham and Women's Hospital, and Harvard Medical School, Boston, Mass.

This work was funded by the International Mesothelioma Program (https://www. brighamandwomens.org/lung-center/diseases-and-conditions/mesothelioma).

Received for publication Jan 25, 2020; revisions received Jan 25, 2020; accepted for publication Feb 29, 2020; available ahead of print May 29, 2020.

Address for reprints: Raphael Bueno, MD, Division of Thoracic Surgery, Brigham and Women's Hospital, 75 Francis St, Boston, MA 02115 (E-mail: rbueno@ bwh.harvard.edu).

J Thorac Cardiovasc Surg 2020;160:1078-83 $0022-5223 / \$ 36.00$

Copyright $₫ 2020$ Published by Elsevier Inc. on behalf of The American Association for Thoracic Surgery

https://doi.org/10.1016/j.jtcvs.2020.02.141

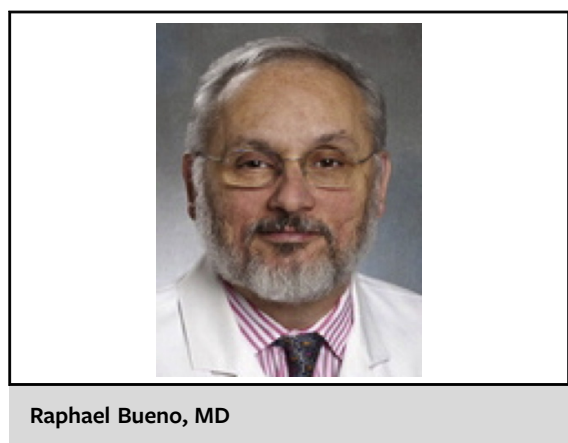

CENTRAL MESSAGE

NGS studies, including the 2018 TCGA publication on MPM, have improved our molecular and genetic understanding of the disease.

This Invited Expert Opinion provides a perspec tive on the following paper: Cancer Discov. 2018;8(12):1548-1565 8290.CD-18-0804.

See Commentaries on pages 1084 and 1086.

MPM is eloquently presented by the Brigham group and will inspire the discovery and translation of novel molecular targets by mesothelioma investigators and practitioners.

\section{Bryan M. Burt, MD}

The-omics technologies have expanded widely because of advances in both mass spectrometry and nucleotide sequencing technology. Together, these approaches have led to numerous new insights in medicine and biology. In cancer, the developments in nucleotide sequencing have been transformative, allowing cancer biologists to characterize cancer-specific driver genes (genomics), identify altered gene expression (transcriptomics, methylomics, interactomics), and generate candidates for novel drug targets. Malignant pleural mesothelioma (MPM) research has also benefitted from those advances. In The Cancer Genome Atlas (TCGA) article "Integrative Molecular Characterization of Malignant Pleural Mesothelioma," published in Cancer Discovery in 2018, Hmeljak and 
colleagues ${ }^{1}$ applied these technologies in an integrated fashion. Before further discussion of the article by TCGA, the history and fundamentals of both MPM and nucleotide sequencing technology merit some introduction.

\section{KEY FEATURES OF MALIGNANT PLEURAL MESOTHELIOMA}

MPM is a deadly, but relatively rare malignancy caused by prior asbestos exposure. MPM is derived from malignant transformation of the mesothelium, the monolayer of epithelial cells lining the pleural lumen. ${ }^{2}$ Approximately $60 \%$ of MPMs are epithelioid, another $20 \%$ are sarcomatoid characterized by spindle cell morphology, and the remaining $20 \%$ are a mixed type called "biphasic mesothelioma." prognostic. The ranges of median survival for patients presenting with sarcomatoid-, biphasic-, and epithelioidtype MPM are 7 to 18 months, 8 to 21 months, and 12 to 27 months, respectively. ${ }^{2}$ Despite the phenotypic and prognostic diversity of these 3 histologic subtypes, few specific differences in genetic etiology have been identified to date.

Several investigations suggest that decades of chronic inflammation in the pleural microenvironment facilitate carcinogenesis. ${ }^{2}$ Asbestos exposure is the initiator of this chronic, carcinogenic proinflammatory milieu in the majority of MPM cases. ${ }^{3}$ Radiation may be implicated in a minority of cases. ${ }^{4}$ Variants in the gene BAPl have also been associated with familiar and sporadic MPM. ${ }^{2}$ Because little is known about the pathogenesis of MPM, the identification of somatic mutations and subsequently altered pathways that drive mesothelial transformation are of great interest in the field. The various "-omics" technologies, especially transcriptomics and genomics, have contributed greatly to our understanding of relevant genetic alterations and pathway perturbations in MPM.

\section{A BRIEF HISTORY OF NEXT-GENERATION SEQUENCING}

The technology allowing whole genome and transcriptome study represents a convergence of numerous discoveries and innovations in molecular biology. In 1953 when Watson and Crick $^{5}$ identified the double-helix chemical structure of DNA, the inevitable quest to sequence and understand the genome began. Rapid, although low throughput, techniques such as Maxam and Gilbert's method $^{6}$ and Sanger sequencing ${ }^{7}$ were essential to fulfilling this mission. The advent of polymerase chain reaction in $1988^{8}$ expedited the rate of gene discoveries, which culminated in 2004 when the entire human genome was finally sequenced. ${ }^{?}$

Soon after, technologies collectively called "next-generation sequencing" (NGS) became available for genomewide study. ${ }^{10}$ These massively parallel sequencing technologies made possible the simultaneous sequencing of millions to trillions of parallel base pairs and dramatically decreased the time and cost of sequencing. The field accelerated as sequencing individual genomes became orders of magnitude cheaper and faster. The thousand genomes project was completed in 2012. ${ }^{11}$ NGS technology was also used to understand functional elements. Whole transcriptomes of tissue were analyzed by sequencing cDNA, so-called RNA sequencing. ${ }^{12}$ In ChIP-seq, chromatin immune precipitation of histone modifications and transcription factors enriched for DNA associated with these macro-molecules and the resulting DNA was sequenced. ${ }^{13}$ The ENCODE project combined these functional approaches, and the project's phase 3 conclusion in 2011 represented a great leap forward in decoding the fundamental language of gene expression and regulation. ${ }^{14}$ After these achievements, costs have continued to decline; individual genomes and transcriptomes are routinely sequenced (Figure 1). Multi-institutional teams such as TCGA, and even individual laboratories, can now afford to tackle individual biological problems by applying multiple NGS approaches to numerous samples. In this way, in the last few years the understanding of MPM has improved because of high-throughput studies.

\section{GENOMICS IN MALIGNANT PLEURAL MESOTHELIOMA BEFORE THE CANCER GENOME ATLAS}

Numerous cytogenetic, molecular genetics, and highdensity array methods have identified many of the specific chromosomal rearrangements, epigenetic alterations, and genes that are important in MPM. ${ }^{15}$ Chromosomal abnormalities have long been recognized as important in MPM, and aneuploidy has been observed to be characterized by widespread chromosomal loss. ${ }^{15}$ These losses frequently occur in regions 1p, 3p14-p21, whole chromosome 4, 6q, $9 \mathrm{p}$, and $22 \mathrm{q} .{ }^{15}$ Notably, several tumor suppressor genes (TSGs) frequently mutated in MPM, cyclin-dependent kinase inhibitor $2 \mathrm{~A}(C D K N 2 A)$, ubiquitin carboxylterminal hydroxylase $(B A P 1)$, and tumor protein $\mathrm{p} 53$ (TP53) are found within 9p21.3, 3p21, and $17 \mathrm{p} 13$, respectively. ${ }^{15}$ In epigenetic studies, tumor suppressors $C D K N 2 A$ and cyclin-dependent kinase inhibitor 2B $(C D K N 2 B)$, among others, were hypermethylated in patients with MPM compared with asbestos-exposed patients who were in turn hypermethylated compared with nonexposed patients. ${ }^{15}$ Loss of TSGs was found to be a common feature in MPM; specific mutations in known TSGs, such as neurofibromatosis type 2 (NF2), large tumor suppressor kinase 2 (LATS2), CDKN2A,CDKN2B, and TP53, were identified mutations in MPM. ${ }^{15,16}$ No oncogene has been identified in MPM to date. ${ }^{15}$ Taken together, these observations suggest MPM is a malignancy resulting from inhibition of cellular mechanisms of tumor 


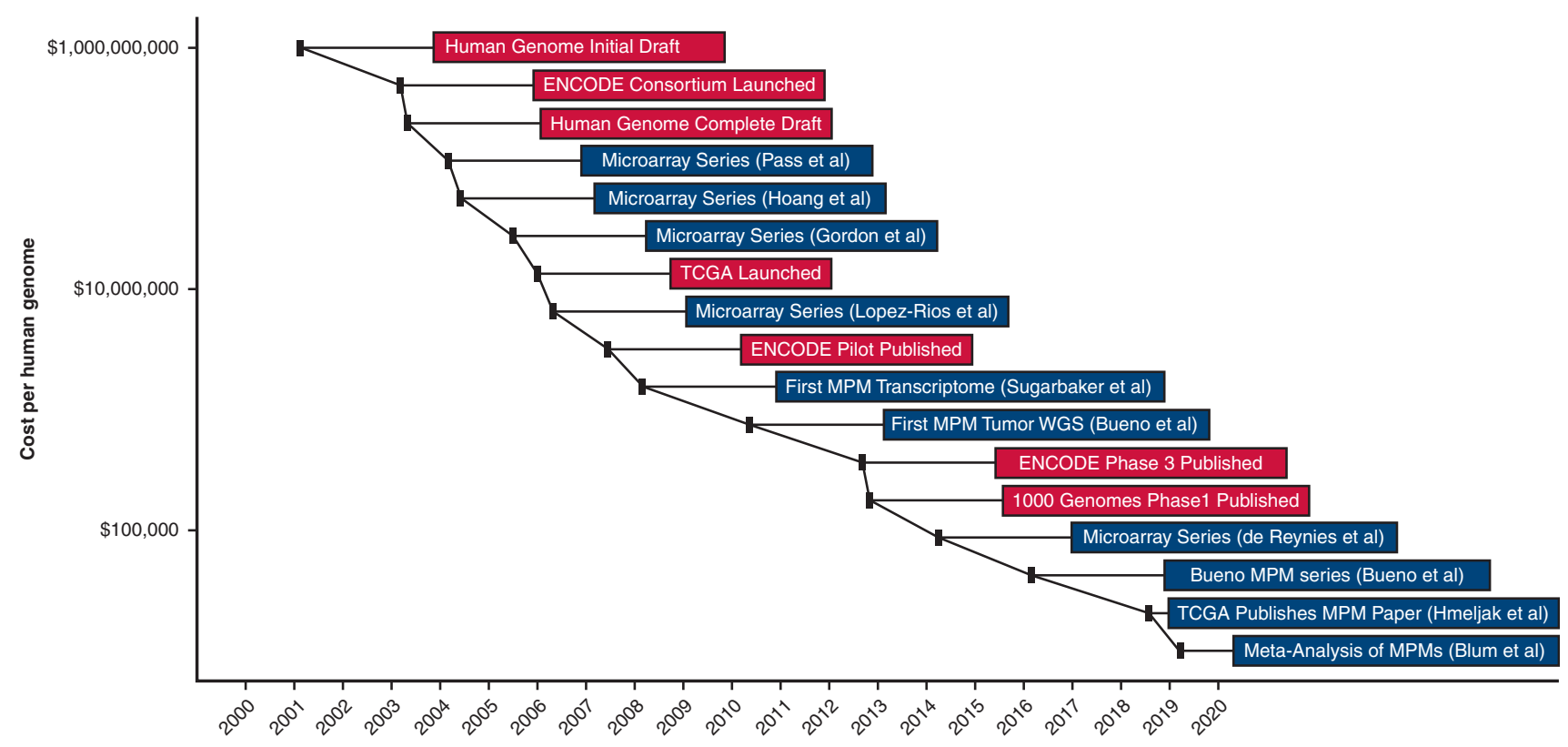

FIGURE 1. NGS experiments in MPM in the context of sequencing costs. Line plot depicts the cost of sequencing a single human genome in USD from data provided by the National Human Genome Research Institute (https://www.genome.gov/about-genomics/fact-sheets/DNA-Sequencing-Costs-Data). Plots are annotated with major landmark achievements in genomics (red) and specific sequencing and microarray experiments in MPM (blue). Appendix E1 shows references of displayed events.

suppression rather than transformation by activation of oncogenes.

In 2016, our group confirmed many of these findings using Illumina SNP arrays, whole exome, and targeted deep sequencing of 95, 99, and 103 tumors, respectively. ${ }^{17}$ Consistent with previous studies, ${ }^{15}$ somatic copy number alterations (SCNAs) identified in the cohort of 95 MPMS were broadly characterized by copy number loss in TSGs (eg, BAP1, NF2, CDKN2B, and TP53). ${ }^{17}$ A subset of MPMs were characterized by copy number neutral widespread loss of heterozygosity. ${ }^{17}$ The previously described tumor suppressors, NF2, BAP1, and TP53 were among the most frequently and significantly mutated in the MPM cohort. ${ }^{17}$ In addition to these, we observed several new significantly mutated genes including histone modifiers, such as SETD2, SETDB1, and SETD5, RNA helicase family members $D D X 3 X$ and $D D X 51$, a target of mTOR negative regulation $U L K 2$, and a calcium channel component $R Y R 2 .^{17}$

\section{TRANSCRIPTOMICS IN MALIGNANT PLEURAL MESOTHELIOMA BEFORE THE CANCER GENOME ATLAS}

Early work in MPM transcriptomics used microarray technology. In 2005, Gordon and colleagues ${ }^{18}$ identified 2 molecular phenotype groups and observed that these groups associated with histologic classification consistent with similar observations in a smaller cohort made by Hoang and colleagues in 2004. ${ }^{19}$ Although the histology of mesothelioma did not perfectly correlate with these molecular features, a spectrum of histologically associated molecular signatures was beginning to emerge. In 2014, de Reyniès and colleagues ${ }^{20}$ profiled 38 MPM primary cell lines using microarray profiling. Clustering of these expression profiles identified 2 major groups. ${ }^{20}$ These clusters were associated with, but not identical to, histology; both groups contained epithelioid samples, but all the sarcomatoid samples were found in cluster $2 .{ }^{20}$ Furthermore, genes associated with epithelioid-mesenchymal transition (EMT) were differentially regulated between the 2 clusters, and cluster 2 exhibited a more mesenchymal phenotype. ${ }^{20}$ This work suggested that a primary feature of MPM heterogeneity was related to EMT phenotype. By using a 3 gene molecular test based on the observed gene expression differences in the 2 clusters, the relationship between the EMT status and histology and survival was demonstrated in 108 MPM tumor samples. ${ }^{20}$

Following this work, in 2016 our group published RNA-sequencing profiles of 211 MPM tumor samples. ${ }^{17}$ In our cohort, unsupervised consensus clustering identified 4 molecular clusters approximately associated with the spectrum from epithelioid to sarcomatoid histology. ${ }^{17}$ Analysis of genes differentially expressed between the 2 extreme clusters identified in our MPM cohort suggested these clusters appeared to be related to EMT consistent with the observations of de Reyniès and colleagues. ${ }^{17,20}$ Additionally, a simple ratio of 2 genes, CLDN15 and VIM, the ratio of CLDN15/VIM 
genes $(\mathrm{C} / \mathrm{V})$ score, significantly discriminated each molecular cluster. ${ }^{17}$

\section{THE CANCER GENOME ATLAS: AN INTEGRATIVE APPROACH IN MALIGNANT PLEURAL MESOTHELIOMA}

Hmeljak and colleagues ${ }^{1}$ performed a comprehensive analysis of 74 MPM tumors as part of the effort by TCGA. Genomic, epigenomic, and transcriptomic profiles were generated using high-throughput array and NGS experiments. Specifically, copy-number arrays and exome sequencing characterized chromosomal and genetic aberrations. For gene expression analysis, TCGA performed total RNA sequencing including long noncoding RNA analysis. In addition, micro-RNA, DNA methylation, and protein level profiles were also obtained using micro-RNA sequencing, hybridization arrays, and reverse-phase protein arrays, respectively.

The SCNA landscape of MPM tumors described in TCGA cohort was consistent with previous NGS results in MPM. ${ }^{1,15,17}$ Several focal and arm-level deletions, but no recurring amplifications, were observed in this cohort. ${ }^{1}$ Loss of regions containing TSGs previously described in MPM such as $C D K N 2 A$ and NF2 were present in 41 $(56 \%)$ and $55(74 \%)$ of 74 MPM tumors, respectively. ${ }^{1}$ Intriguingly, genomic analysis revealed a subpopulation of MPM tumors ( $\mathrm{n}=3$ in TCGA) with loss of heterozygosity across more than $80 \%$ of the genome. ${ }^{1}$ This subpopulation was also observed in our previous study. ${ }^{17}$ To better describe this cohort, the 3 TCGA loss of heterozygosity samples were combined with 2 additional cases from the Japanese International Cancer Genome Consortium for a total of $5(3 \%)$ samples of 154 MPM tumors examined. ${ }^{1}$ This subpopulation was defined as having "genomic-near haploidization," where homozygous deletion of SETDB1 was a common feature of this subgroup, but absent in all other samples. ${ }^{1}$ Interestingly, 4 of these samples were female and 4 of them had TP53 mutations.

Genomic alterations at the single nucleotide level were also consistent with previous work. ${ }^{1,15,17}$ The TSGs, BAP1, NF2, TP53, LATS2, and SETD2 were mutated significantly above the background mutation rate in this cohort. ${ }^{1}$ Several mutations identified in previous NGS studies ${ }^{17}$ including SETDB1, DDX3X, DDX51, ULK2, and $R Y R 2$, were found to be recurrently mutated in this cohort, but did not rise to significance likely because of a lack of statistical power. As in previous MPM studies. ${ }^{15,17}$ these mutations in TSGs were characterized by inactivation. Although BAPl genetic alterations displayed nonrandom patterns of co-occurrence with recurrently mutated genes (NF2, TP53, LATS2, SETD2, and $M A L A T 1)$, the associations were not significant when multiple hypothesis testing was taken into account. ${ }^{1} \mathrm{Tu}-$ mors with BAPl homozygous mutation had significantly more arm stability when compared with wild-type tumors. ${ }^{1}$

In this study, SCNAs, gene expression, and epigenetic information were integrated using 2 previously published clustering algorithms, iCluster ${ }^{21}$ and PARADIGM. ${ }^{22}$ Both algorithms identified 4 distinct groups of MPMs. ${ }^{1}$ There was strong concordance in the grouping of samples between the 2 methods, especially for cluster 1 and cluster 4 from each clustering. ${ }^{1}$ In both analyses, the clusters were significantly associated with histology, with cluster 1 enriched for epithelioid tumors and cluster 4 enriched for sarcomatoid tumors as observed in other studies. ${ }^{1,17,20}$ In addition, EMT genes were found to be differentially expressed between clusters 1 and $4{ }^{1}$ These results together with previous work ${ }^{17,20}$ indicate that molecular data identify distinct groups of MPM independent of algorithm or cohort. TCGA clusters were also characterized by distinct immunological cell infiltrates. ${ }^{1}$ VISTA, an immune checkpoint inhibitor, was found to be highly expressed in cluster 1 compared with the other clusters. ${ }^{1}$ These findings are suggestive of distinct immune microenvironments in different groups of MPMs.

\section{SINCE THE CANCER GENOME ATLAS: AN UPDATE IN TRANSCRIPTOMICS}

More recently, a comprehensive meta-analysis of mesothelioma profiles has been conducted by Blum and colleagues. $^{23}$ Previously published data from 2 RNA sequencing ${ }^{1,17}$ and 3 microarray ${ }^{18,20,24}$ MPM expression profiles were analyzed with 63 new microarray profiles of primary MPM. In this analysis, Blum and colleagues characterized the molecular profiles of MPM samples from multiple studies. First, they identified 4 distinct groups (C1A, $\mathrm{C} 1 \mathrm{~B}, \mathrm{C} 2 \mathrm{~A}$, and $\mathrm{C} 2 \mathrm{~B}$ ) in their $63 \mathrm{MPM}$ samples, and then the expression profile of these groups was compared with clusters of previously published MPM expression data. ${ }^{1,17,18,20,24}$ This analysis identified 2 main groups of highly correlated clusters present in all datasets that corresponded to the most extreme epithelioid and sarcomatoid phenotypes. ${ }^{23}$ The remaining tumors did not show high correlation, indicating that other clusters may represent various thresholds applied to a continuum, or "histo-molecular gradient." Next, by using a deconvolution approach, 2 molecular signatures, E-score and S-score, were identified to define the proportion of epithelioid-like and sarcomatoidlike components in each tumor. Both scores are composed of the same 150 genes, but the contribution of each gene to the E- or S-score is different.

We previously reported that the ratio of CLDN15 and $V I M(\mathrm{C} / \mathrm{V}$ score) significantly discriminates among the 4 transcriptional clusters in our cohort. ${ }^{17}$ To determine whether the $\mathrm{C} / \mathrm{V}$ score could be an effective metric for describing "EMT-ness" for each MPM tumor, we examined the correlation between the $\mathrm{C} / \mathrm{V}$ score and 3 other published 
metrics associated with EMT: E-score, ${ }^{23}$ S-score, ${ }^{23}$ and Creighton score $\left(\mathrm{EMT}_{\text {Creighton }}\right){ }^{25}$ The $\mathrm{EMT}_{\text {Creighton }}$ score, consisting of 16 genes, was previously used to analyze EMT-associated genes in molecular profiling datasets of breast and lung tumors. ${ }^{25}$ We used the RNA-seq profiles from TCGA ${ }^{1}$ and our cohort ${ }^{17}$ in addition to the microarray profiles from the series by Blum and colleagues. ${ }^{23} \mathrm{We}$ found that the $\mathrm{C} / \mathrm{V}$ score was significantly correlated with all 3 EMT metrics scores (Figure 2). This suggested that the $\mathrm{C} /$ $\mathrm{V}$ score may be an alternative metric for determining the "EMT-component" in each MPM tumor using only 2 genes.
The existence of a "histomolecular gradient" associated with EMT across MPM tumors has been identified by several studies suggesting that each MPM is a mixture of epitheliod and sarcomatoid components that may define the molecular profile of each tumor.

\section{TOWARD THE FUTURE}

The genomic and transcriptomic work completed before, during, and after TCGA study has thus far focused on the heterogeneity across different tumors. We now know the prevalent genomic alterations in MPM at both the gene
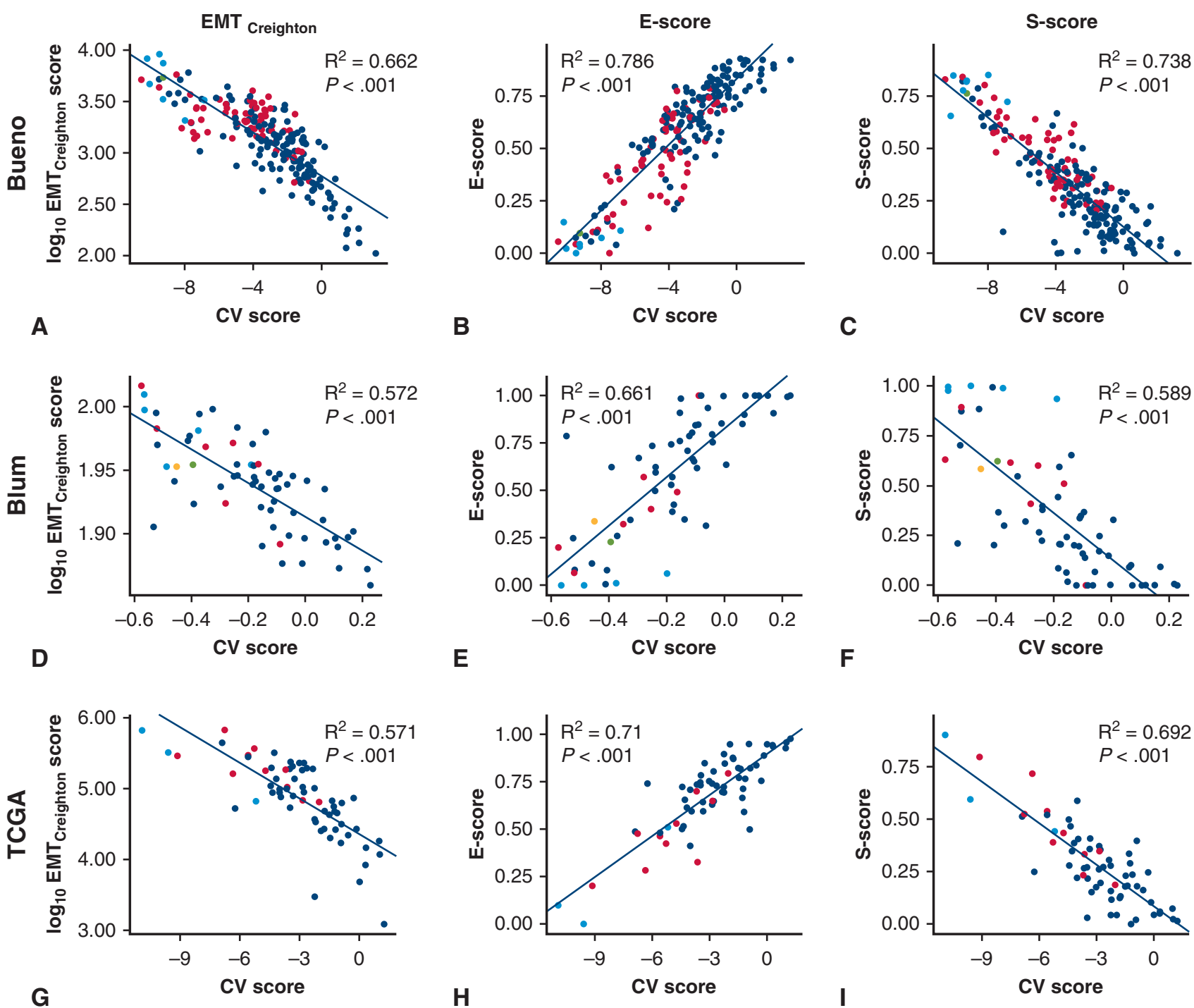

Histology epithelioid biphasic desmoplastic lymphohistiocytoid sarcomatoid

FIGURE 2. $\mathrm{C} / \mathrm{V}$ score correlates with E-score, S-score, and $\mathrm{EMT}_{\text {Creighton }}$ score. Scatterplots depict correlation among the C/V score ( $x$-axis) and 3 EMT metrics (y-axis), that is, the $\mathrm{EMT}_{\text {Creighton }}(\mathrm{A}, \mathrm{D}, \mathrm{G})$, the E-score (B, E, H), S-Score (C, F, I), in the Bueno RNA-seq (A-C), Blum microarray (D-F), and TCGA RNA-seq (G-I) MPM series. The line in each plot illustrates the least squares fit, and the $\mathrm{R}^{2}$ and $P$ values for the Pearson correlation between $\mathrm{C} / \mathrm{V}$ score and the respective EMT metric for each series are displayed within the relevant panel. $E M T_{C r e i g h t o n}, 16$ gene score published by Creighton and colleagues; $C / V$, ratio of CLDN15/VIM genes; TCGA, The Cancer Genome Atlas. 
and chromosome levels. We have also learned from RNA-sequencing and micro-array studies that the heterogeneity across MPM tumors exists on a "histomolecular gradient" that significantly correlates with EMT. This gradient is prognostic and can be described effectively using the $\mathrm{C} / \mathrm{V}$ score. Despite these insights into the heterogeneity across tumors, little is understood about the heterogeneity within MPM tumors. MPMs are typically bulky with large surface area; different regions of these large tumors could have distinct histomolecular phenotypes. Moreover, many MPMs are biphasic with admixtures of cell phenotypes. Therefore, genomic and transcriptomic heterogeneity studies that examine samples across multiple sites within a patient and use single-cell sequencing techniques or both will be of particular interest for future work.

\section{Conflict of Interest Statement}

The authors reported no conflicts of interest.

The Journal policy requires editors and reviewers to disclose conflicts of interest and to decline handling or reviewing manuscripts for which they may have a conflict of interest. The editors and reviewers of this article have no conflicts of interest.

\section{References}

1. Hmeljak J, Sanchez-Vega F, Hoadley KA, Shih J, Stewart C, Heiman D, et al. Integrative molecular characterization of malignant pleural mesothelioma. Cancer Discov. 2018;8:1548-65.

2. Yap TA, Aerts JG, Popat S, Fennell DA. Novel insights into mesothelioma biology and implications for therapy. Nat Rev Cancer. 2017;17:475-88.

3. Peto J, Seidman H, Selikoff I. Mesothelioma mortality in asbestos workers: implications for models of carcinogenesis and risk assessment. Br J Cancer. 1982; 45:124-35.

4. van Kaick G, Dalheimer A, Hornik S, Kaul A, Liebermann D. The German thorotrast study: recent results and assessment of risks. Radiat Res. 1999;152: S64.

5. Watson JD, Crick FHC. Molecular structure of nucleic acids: a structure for deoxyribose nucleic acid. Nature. 1953;171:737-8.

6. Maxam A, Gilbert W. A new method for sequencing DNA. Proc Natl Acad Sci. 1977;74:560-4

7. Sanger F, Coulson AR. A rapid method for determining sequences in DNA by primed synthesis with DNA polymerase. J Mol Biol. 1975;94:441-8.
8. Saiki R, Gelfand D, Stoffel S, Higuchi R. Primer-directed enzymatic amplification of DNA with a thermostable DNA polymerase. Science. 1988;239:487-91.

9. International Human Genome Sequencing Consortium. Finishing the euchromatic sequence of the human genome. Nature. 2004;431:931-45.

10. Tucker T, Marra M, Friedman JM. Massively parallel sequencing: the next big thing in genetic medicine. Am J Hum Genet. 2009;85:142-54.

11. The 1000 Genomes Project Consortium. An integrated map of genetic variation from 1,092 human genomes. Nature. 2012;491:56-65.

12. Wang Z, Gerstein M, Snyder M. RNA-Seq: a revolutionary tool for transcriptomics. Nat Rev Genet. 2009;10:57-63.

13. Mikkelsen TS, Ku M, Jaffe DB, Issac B, Lieberman E. Genome-wide maps of chromatin state in pluripotent and lineage-committed cells. Nature. 2007;448: 553-60.

14. The ENCODE Project Consortium. An integrated encyclopedia of DNA elements in the human genome. Nature. 2012;489:57-74.

15. Jean D, Daubriac J, Pimpec-Barthes F, Galateau-Salle F, Jaurand M-C. Molecular changes in mesothelioma with an impact on prognosis and treatment. Arch Pathol Lab Med. 2012;136:277-93.

16. Murakami H, Mizuno T, Taniguchi T, Fujii M, Ishiguro F. LATS2 is a tumor suppressor gene of malignant mesothelioma. Cancer Res. 2011;71: 873-83.

17. Bueno R, Stawiski EW, Goldstein LD, Durinck S, De Rienzo A, Modrusan Z et al. Comprehensive genomic analysis of malignant pleural mesothelioma identifies recurrent mutations, gene fusions and splicing alterations. Nat Genet. 2016; 48:407-16.

18. Gordon GJ, Rockwell GN, Jensen RV, Rheinwald JG, Glickman JN. Identification of novel candidate oncogenes and tumor suppressors in malignant pleural mesothelioma using large-scale transcriptional profiling. Am J Pathol. 2005;166: 1827-40.

19. Hoang CD, D'Cunha J, Kratzke MG, Casmey CE, Frizelle SP. Gene expression profiling identifies matriptase overexpression in malignant mesothelioma. Chest 2004; $125: 1843-52$

20. de Reyniès A, Jaurand M-C, Renier A, Couchy G, Hysi I. Molecular classification of malignant pleural mesothelioma: identification of a poor prognosis subgroup linked to the epithelial-to-mesenchymal transition. Clin Cancer Res 2014;20:1323-34

21. Shen R, Olshen AB, Ladanyi M. Integrative clustering of multiple genomic data types using a joint latent variable model with application to breast and lung cancer subtype analysis. Bioinformatics. 2009;25:2906-12.

22. Vaske CJ, Benz SC, Sanborn ZJ, Earl D, Szeto C. Inference of patient-specific pathway activities from multi-dimensional cancer genomics data using PARADIGM. Bioinformatics. 2010;26:i237-45.

23. Blum Y, Meiller C, Quetel L, Elarouci N, Ayadi M, Tashtanbaeva D, et al. Dissecting heterogeneity in malignant pleural mesothelioma through histo-molecular gradients for clinical applications. Nat Commun. 2019;10: 1333

24. López-Ríos F, Chuai S, Flores R, Shimizu S, Ohno T. Global gene expression profiling of pleural mesotheliomas: overexpression of aurora kinases and P16/ CDKN2A deletion as prognostic factors and critical evaluation of microarraybased prognostic prediction. Cancer Res. 2006;66:2970-9.

25. Creighton CJ, Gibbons DL, Kurie JM. The role of epithelial-mesenchymal transition programming in invasion and metastasis: a clinical perspective. Cancer Manag Res. 2013;5:187-95. 


\section{APPENDIX E1}

\begin{tabular}{|c|c|}
\hline Event & Reference \\
\hline Human Genome Initial Draft & $\begin{array}{l}\text { International Human Genome Sequencing Consortium. Initial sequencing and analysis of } \\
\text { the human genome. Nature. 2001;409:860-921. } \\
\text { Venter JC, Adams MD, Myers EW, Li PW, Mural RJ, Sutton GG, et al. The sequence of the } \\
\text { human genome. Science. 2001;291:1304-51. }\end{array}$ \\
\hline ENCODE Consortium Launched & $\begin{array}{l}\text { The ENCODE Project Consortium. The ENCODE (ENCyclopedia Of DNA Elements) } \\
\text { Project. Science. 2004;306:636-40. https://videocast.nih.gov/watch=2306 }\end{array}$ \\
\hline Human Genome Complete Draft & $\begin{array}{l}\text { International Human Genome Sequencing Consortium. Finishing the euchromatic } \\
\text { sequence of the human genome. Nature. 2004;431:931-45. }\end{array}$ \\
\hline Microarray Series (Pass and colleagues) & $\begin{array}{l}\text { Pass HI, Liu Z, Wali A, Bueno R, Land S, Lott D, et al. Gene expression profiles predict } \\
\text { survival and progression of pleural mesothelioma. Clin Cancer Res. 2004;10:849-59. }\end{array}$ \\
\hline Microarray Series (Hoang and colleagues) & $\begin{array}{l}\text { Hoang CD, D’Cunha J, Kratzke MG, Casmey CE, Frizelle SP. Gene expression profiling } \\
\text { identifies matriptase overexpression in malignant mesothelioma. Chest. 2004;125:1843- } \\
52 .\end{array}$ \\
\hline Microarray Series (Gordon and colleagues) & $\begin{array}{l}\text { Gordon GJ, Rockwell GN, Jensen RV, Rheinwald JG, Glickman JN. Identification of novel } \\
\text { candidate oncogenes and tumor suppressors in malignant pleural mesothelioma using } \\
\text { large-scale transcriptional profiling. Am J Pathol. 2005;166:1827-40. }\end{array}$ \\
\hline TCGA Launched & $\begin{array}{l}\text { https://www.cancer.gov/about-nci/organization/ccg/research/structural-genomics/tcga/ } \\
\text { history }\end{array}$ \\
\hline Microarray Series (López-Ríos and colleagues) & $\begin{array}{l}\text { López-Ríos F, Chuai S, Flores R, Shimizu S, Ohno T. Global gene expression profiling of } \\
\text { pleural mesotheliomas: overexpression of aurora kinases and P16/CDKN2A deletion as } \\
\text { prognostic factors and critical evaluation of microarray-based prognostic prediction. } \\
\text { Cancer Res. 2006;66:2970-9 }\end{array}$ \\
\hline ENCODE Pilot Published & $\begin{array}{l}\text { The ENCODE Project Consortium. Identification and analysis of functional elements in } \\
1 \% \text { of the human genome by the ENCODE pilot project. Nature. } 2007 ; 447: 799-816 \text {. }\end{array}$ \\
\hline First MPM Transcriptome (Sugarbaker and colleagues) & $\begin{array}{l}\text { Sugarbaker DJ, Richards WG, Gordon GJ, Dong L, De Rienzo A, Maulik G, et al. } \\
\text { Transcriptome sequencing of malignant pleural mesothelioma tumors. Proc Natl Acad } \\
\text { Sci. 2007;105:3521-6. }\end{array}$ \\
\hline First MPM Tumor WGS (Bueno and colleagues) & $\begin{array}{l}\text { Bueno R, De Rienzo A, Dong L, Gordon GJ, Hercus CF, Richards WG, et al. Second } \\
\text { generation sequencing of the mesothelioma tumor genome. PLoS One. 2010;5:e10612. }\end{array}$ \\
\hline 1000 Genomes Pilot Published & $\begin{array}{l}\text { The } 1000 \text { Genomes Project Consortium. A map of human genome variation from } \\
\text { population-scale sequencing. Nature. 2010;467:1061-73. }\end{array}$ \\
\hline ENCODE Phase 3 Published & $\begin{array}{l}\text { The ENCODE Project Consortium. An integrated encyclopedia of DNA elements in the } \\
\text { human genome. Nature. 2012;489:57-74. } \\
\text { The ENCODE Project Consortium. A User's Guide to the Encyclopedia of DNA Elements } \\
\text { (ENCODE). PLoS Biol. 2011;9:e1001046. }\end{array}$ \\
\hline 1000 Genomes Phase 1 Published & $\begin{array}{l}\text { The } 1000 \text { Genomes Project Consortium. An integrated map of genetic variation from } 1092 \\
\text { human genomes. Nature. 2012;491:56-65. }\end{array}$ \\
\hline Microarray Series (de Reyniès and colleagues) & $\begin{array}{l}\text { de Reyniès A, Jaurand M-C, Renier A, Couchy G, Hysi I. Molecular classification of } \\
\text { malignant pleural mesothelioma: identification of a poor prognosis subgroup linked to } \\
\text { the epithelial-to-mesenchymal transition. Clin Cancer Res. 2014;20:1323-34. }\end{array}$ \\
\hline 1000 Genomes Phase 3 Published & $\begin{array}{l}\text { Sudmant PH, Rausch T, Gardner EJ, Handsaker RE, Abyzov A, Huddleston J. An } \\
\text { integrated map of structural variation in } 2504 \text { human genomes. Nature. 2015;526:75-81. } \\
\text { The } 1000 \text { Genomes Project Consortium. A global reference for human genetic variation. } \\
\quad \text { Nature. } 2015 ; 526: 68-74 \text {. }\end{array}$ \\
\hline Bueno MPM series (Bueno and colleagues) & $\begin{array}{l}\text { Bueno R, Stawiski EW, Goldstein LD, Durinck S, De Rienzo A, Modrusan Z, et al. } \\
\text { Comprehensive genomic analysis of malignant pleural mesothelioma identifies recurrent } \\
\text { mutations, gene fusions and splicing alterations. Nat Genet. 2016;48:407-16. }\end{array}$ \\
\hline
\end{tabular}


Event

TCGA Publishes MPM Paper (Hmeljak and colleagues)

Meta-Analysis of MPMs (Blum and colleagues)

\section{Reference}

Hmeljak J, Sanchez-Vega F, Hoadley KA, Shih J, Stewart C, Heiman DI, et al. Integrative molecular characterization of malignant pleural mesothelioma. Cancer Discov. 2018;8:1548-65

Blum Y, Meiller C, Quetel L, Elarouci N, Ayadi M, Tashtanbaeva D, et al. Dissecting heterogeneity in malignant pleural mesothelioma through histo-molecular gradients for clinical applications. Nat Commun. 2019;10:1333. 